\title{
Job-training Support System using Smartphone Virtual Reality
}

\author{
Kenzaburo Miyawaki ${ }^{\mathrm{a},}$, Soichi Okabe ${ }^{\mathrm{a}}$ \\ ${ }^{a}$ Faculty of Information Science and Technology, Osaka Institute of Technology, Hiyakata-City, Osaka-fu, Japan \\ *Corresponding Author: kenzaburo.miyawaki@oit.ac.jp
}

\begin{abstract}
Employees who newly belong to part-time jobs or part-time workers often conduct training to learn business skills and knowledge. However, companies have to pay a lot of costs for the job-trainings, because it needs lecturers, long time and facilities for the training environment. This disadvantage becomes more conspicuous especially in workplaces and industries where people change frequently. Therefore, we propose a job-training support system using Virtual Reality (VR) with the purpose of improving training efficiency and cost reduction. The system uses a smartphone, VR goggles and AR markers to construct a virtual job-training environment, and can recognize actions of job-trainees. By the result of action recognition, the system can show an appropriate instruction of the job-training. Job-trainees can train alone by this system anytime, anywhere.
\end{abstract}

Keywords: virtual reality, smartphone, job-training.

\section{Introduction}

Part-time workers are often trained to learn work skills and knowledge. The company who adopt the workers has to pay a lot of costs for the job training which needs lecturers, long time and facilities for the training environment. This disadvantage becomes more conspicuous especially in workplaces and industries where people change frequently.

Therefore, we propose a system to support training of work procedures by using Virtual Reality (VR) for part-time employees who are newly hired. The system combines computer graphics objects into a real space and enables interaction between the virtual objects and a user. In order to realize the system using $\mathrm{VR}$, it is conceivable to use a Head Mounted Display (HMD). However, HMDs for $\mathrm{VR}^{(1)}$ often require relatively high-performance desktop PCs, and are too expensive to introduce for part-time training purposes.

Therefore, we propose a low cost and easy-to-install job-training support system using VR goggles for smartphones. The smartphone's VR goggles originated from Google Cardboard ${ }^{(2)}$, and very low cost, reasonable and easy method to get Virtual Reality experiences.

As the start of the implementation of smartphone's VR job-training system, we focused on part-time workers for cooking tasks. In general, food industries have very high employment and turnover rates than others, and job-training support for the food service will be useful.

The rest of this paper is organized as follows, In the chapter 2, we describe related works. Chapter 3 shows our system's overview. After that, we explain the methods for implementation of the system in chapter 4. Finally, we conclude this paper in chapter 5 .

\section{Related works}

The research on VR is actively conducted, and since the user can interact with the CG objects, many researches for the purpose of the work support can be seen. Furuya et al., proposes a work support system which can project the viewpoint of a leader who is in a remote place over the HMD of workers ${ }^{(3)}$. However, this research assumes that the human leader teaches instructions to the workers, and a high performance PC and HMD are required.

Cannon IT Solutions Co., Ltd. sells "MREAL" software, which uses HMD and Mixed Reality technologies, as a business support tool for manufacturing industry, but it is very expensive and is very hard to use for ordinal companies like as popular supermarkets.

\section{System overview}

Our goal is realization of a low-cost job-training support system that part-time workers can easily use 
anytime. This system does not require both an expensive VR HMD and a high-performance desktop PC. We use AR markers, smartphones, and low-cost VR goggles that incorporate smartphones.

Fig. 1 shows an overview of the proposed system. A job-trainee sets the smartphone inside of the VR goggles and wears the goggles at the time of training. Then, AR markers are attached on boxes and boards which imitate equipment and objects actually used in the workplace. For example, microwave-open, tray and cooking-ingredients are important objects on the supermarket's kitchen. By looking at the boxes and boards through the smartphone camera built into the goggles, the virtual workplace and equipment can be displayed in front of the job-trainee.

In addition, based on the interaction between the job-trainee and the virtual objects, the system recognizes to which procedure the work is currently progressed, thereby displaying an appropriate instruction of the job-training to be performed next on the screen of the smartphone.

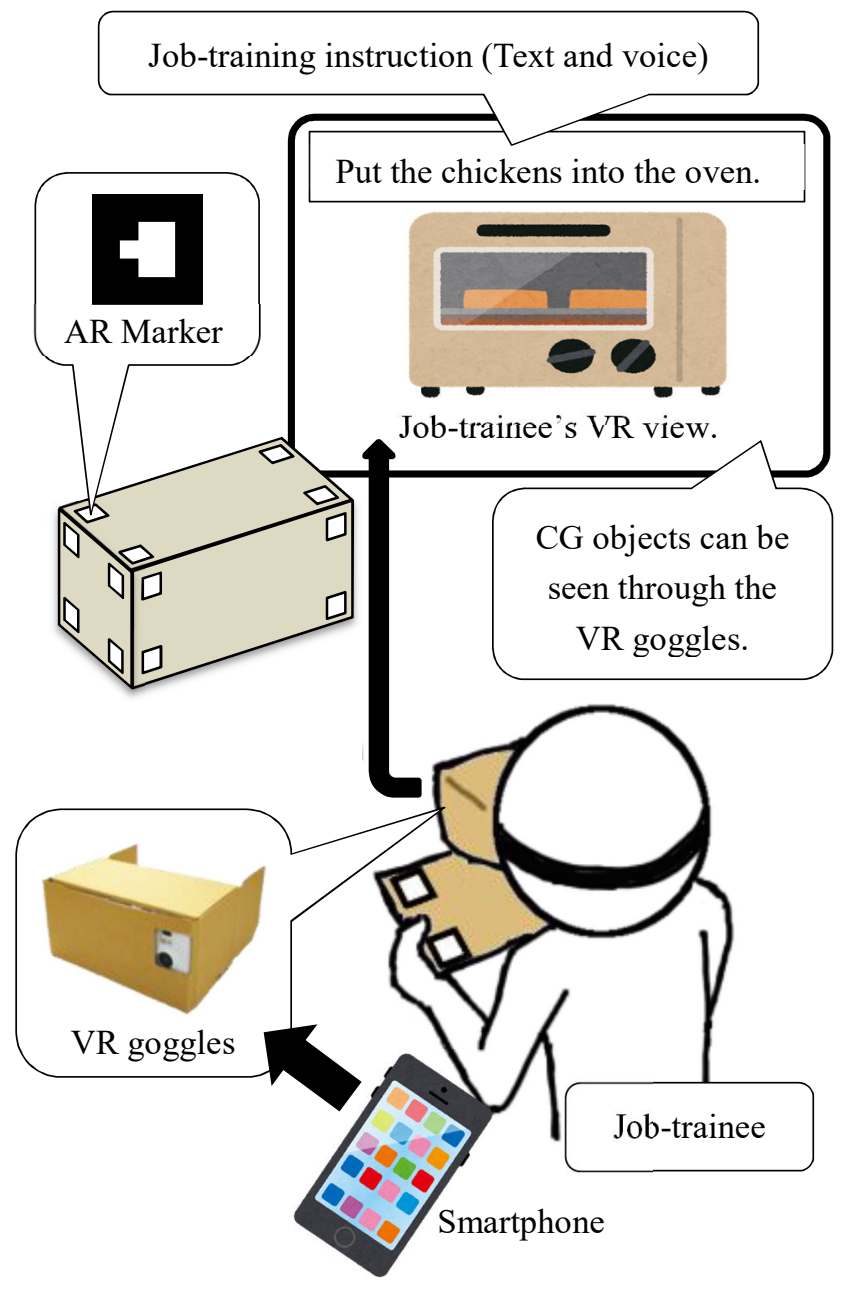

Fig. 1 System overview
This will support the acquisition of skills on a self-directed basis without the need for human job-trainer.

We assume that the boxes and boards which AR markers are pasted on and VR goggles are distributed from companies to employees when they are hired. If part-time employees install this system on their smartphones, they can immediately conduct training, acquire skills, and start work at the actual site.

\section{Implementation}

\subsection{Example system}

As explained at chapter 1, we focused on the job-training support for the food industry, and developed an example system that assumed the cooking task of grilled chicken (Yakitori, in Japanese) in the kitchen of the supermarket. The job-trainee can learn operation procedures of a microwave-oven to cook the chicken.

Sometimes an oven in a supermarket kitchen is different from ordinary household ovens. For example, supermarket kitchen may use a special oven which is customized for specific cooking ingredients, and very old type oven may be used because of shortage of equipment investment. New part-time workers take a long time to learn how to operate that rare ovens. By using this system, job-trainees can lean operation procedure of the equipment anytime.

Furthermore, even if the part-time workers encounter changes of workplace environment, such as relocation, model change of cooking tools and so on, they can easily start the job-training for new situation by changing the configuration of the job-training support system.

\subsection{Building a virtual workplace}

The game engine Unity ${ }^{(5)}$ and the ARToolKit ${ }^{(6)}$ are used for the system development. ARToolKit is applicable to the detection of the AR markers pasted on the box and boards.

The objects used at the supermarket kitchen of grilled chickens is an oven, a tray for carrying food, and grilled chickens. In order to build the virtual objects of kitchen, we create a box and a boards with AR markers attached. Since these materials do not have any problems as long as they have appropriate strength, they can be made extremely inexpensively. We used cardboard because of the good availability in this research.

Fig. 2 to 4 show a box and boards which AR markers 
are attached on, and a virtual oven, tray, and grilled chicken displayed superimposed thereon. The AR markers are $4 \mathrm{~cm}$ squares and we use multi marker recognition, which can group some markers and use the group as one large marker.

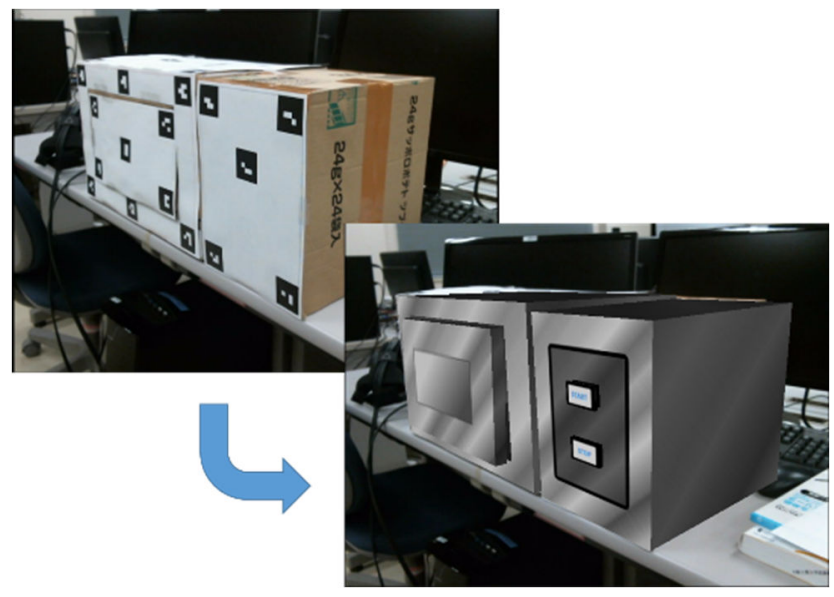

Fig. 2 Virtual oven using multi-marker

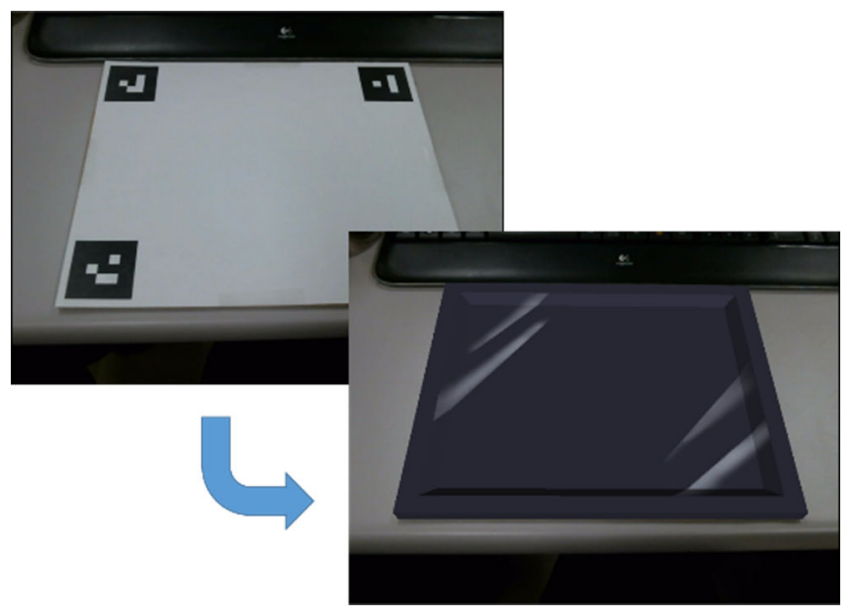

Fig. 3 Virtual baking tray using multi-marker

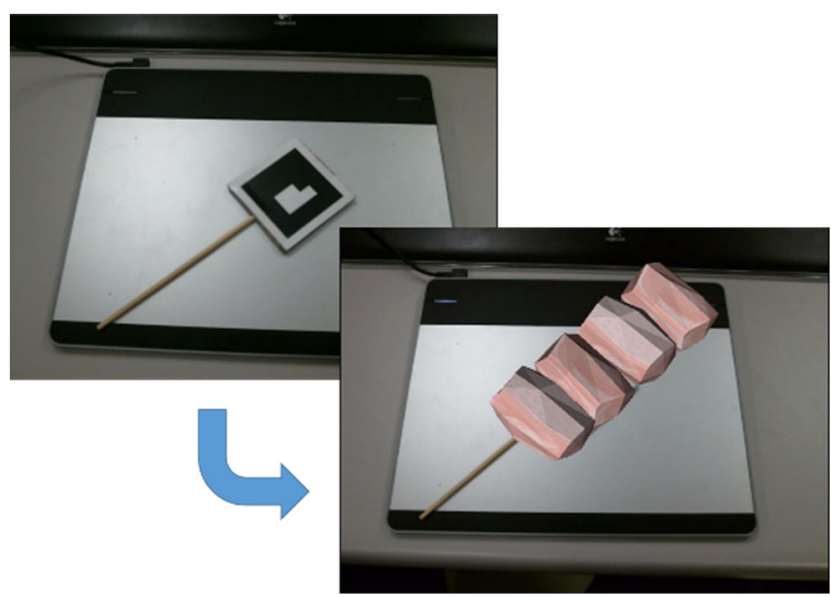

Fig.4 Virtual skewered chicken using a marker

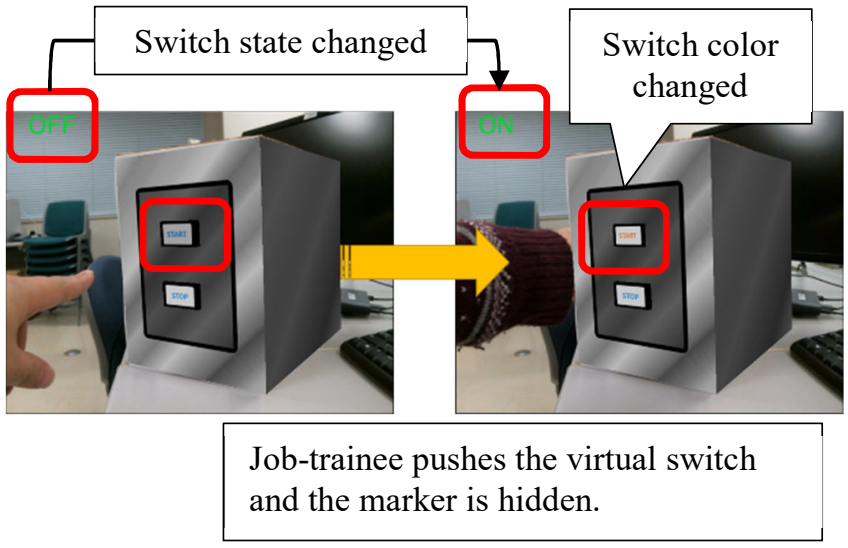

Fig. 5 Operation of virtual switch of oven

This method enhances the robustness of marker recognition. Even if some markers are occluded ARToolKit can recognize the marker group.

\subsection{Interaction with virtual objects}

Since the oven is equipped with a switch, it is necessary to allow the job-trainee to operate the switches installed in the virtual oven. This is implemented by detecting the disappearance of the marker.

Fig. 5 is a portion of the virtual oven including switches. The markers are pasted on the locations of the virtual switches. When the job-trainee pressed a virtual switch, the corresponding AR marker will be lost from the smartphone camera. By checking that, our system understands the press of the switch.

\subsection{Action recognition of the job-trainee}

Fig. 6 shows the state transition model of the grilled chicken cooking. During the job training, the system recognizes which procedure the job-trainee is currently performing, and based on that, informs the job-trainee what to do next. The recognition of the action is performed by the state transition model of Fig. 6. The transitions between each state can be represented by events, such as contact between virtual objects or interaction between the job-trainee and virtual objects.

Contact detection between objects can be easily implemented by the collider, which is one of the functions of Unity. That is used for detection of putting the baking tray in the oven and putting grilled chickens on the baking tray. 


\section{State-1}

Put the skewered chickens on the baking tray.

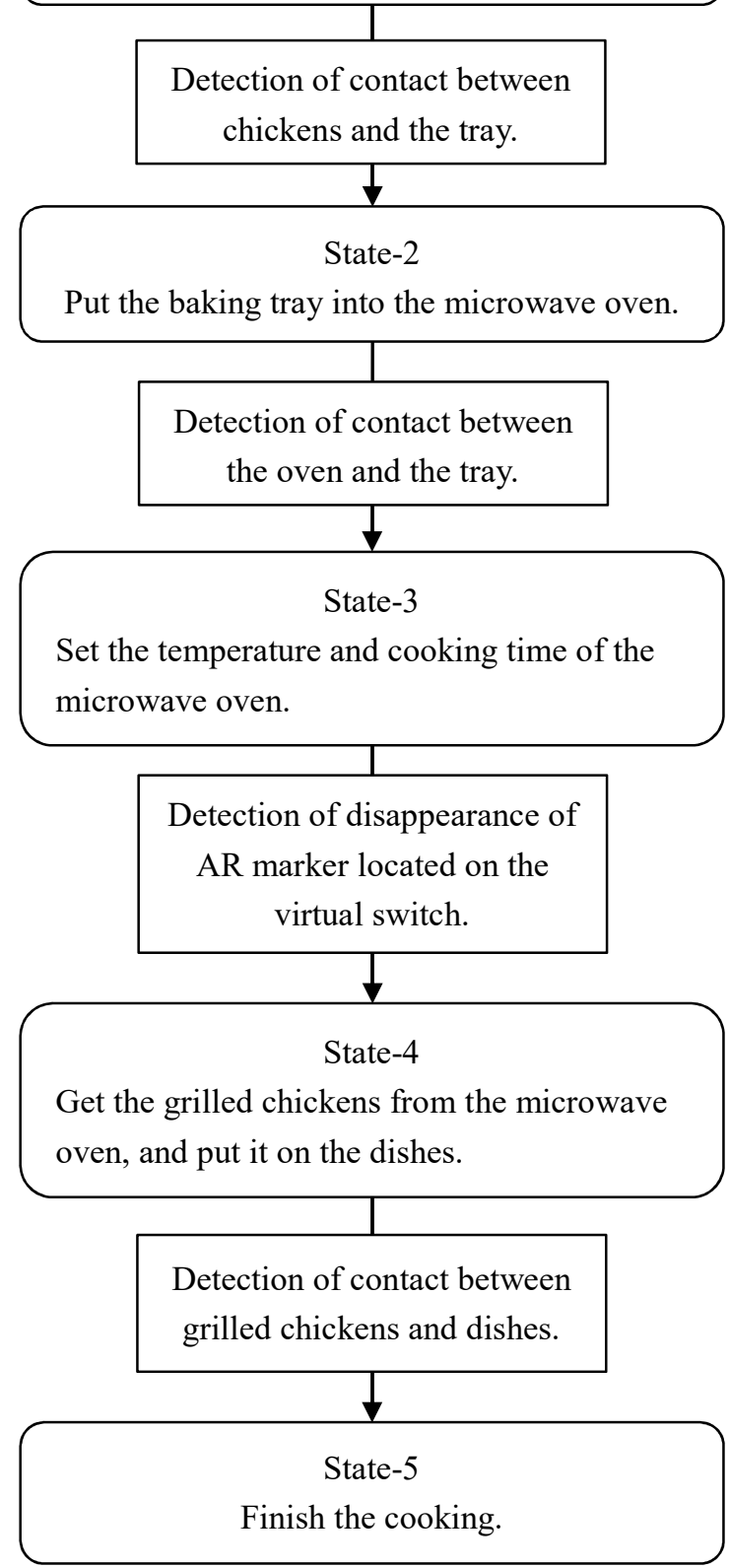

Fig. 6 State transition model of grilled chicken cooking

\section{Conclusion}

In this paper, we proposed a job-training support system for part-time workers using smartphone's VR and AR markers. We focused on the grilled chicken cooking task in the supermarket kitchen, and successfully built the virtual workplace for the job-training. The environment includes virtual object such as an oven, baking-tray, skewered chickens and so on. Job-trainee can see that through smartphone's VR goggles. Furthermore, the system recognizes the job-trainee's action based on the interaction between the virtual objects, and displays the procedure to be performed next, and the job-trainee can learn the work process alone.

For the future work, we are planning to improve our system to realize more realistic job-training. We are challenging to recognize the hands' shape by the smartphone's camera to implements a recognition system of more complex interaction between the job-trainee and virtual objects.

\section{Acknowledgment}

This work was supported by JSPS KAKENHI Grant Number 17K01160.

\section{References}

(1) HTC Corporation: "VIVE Discover Virtual Reality Beyond Imagination", https://www.vive.com/us/

(2) Google: "Google Cardboard - Google VR", https://vr.google.com/cardboard/

(3) Shinnosuke Furuya, Satoru Odera and Kenichi Okada: "The MR Remote Cooperation with Indication by Mutual CG presentation and Afterimages" (in Japanese), Transactions of the Virtual Reality Society of Japan, Vol. 20, No. 4, pp. 283-289, 2015

(4) Canon IT Solutions Inc.: "MR Canon IT Solutions", https://www.canon-its.co.jp/solution/mr/\#anc_op_4

(5) Unity Technologies: "Unity”, https://unity.com/

(6) Hirokazu Kato and Mark Billinghurst: "Marker tracking and hmd calibration for a video-based augmented reality conferencing system", Proc. of the 2nd IEEE and ACM International Workshop on Augmented Reality (IWAR'99), pages 85-94, 1999. 